Review began 01/14/2022 Review ended 01/21/2022 Published 01/25/2022

๑) Copyright 2022

Nathal et al. This is an open access article distributed under the terms of the Creative Commons Attribution License CC-BY 4.0. which permits unrestricted use, distribution, and reproduction in any medium, provided the original author and source are credited.

\section{Subarachnoid Hemorrhage From Rupture of an Undiagnosed Posterior Circulation Aneurysm During Sellar Tumor Surgery}

Edgar Nathal ${ }^{1}$, Rubén Mormandi ${ }^{2}$, Andrés E. Cervio ${ }^{2}$, Juan P. Navarro-Garcia de Llano ${ }^{1}$, Alejandro CejaEspinosa $^{1}$

1. Neurosurgery, Instituto Nacional de Neurología y Neurocirugía Manuel Velasco Suárez, Mexico City, MEX 2. Neurosurgery, Instituto Fleni, Buenos Aires, ARG

Corresponding author: Juan P. Navarro-Garcia de Llano, jpnavarroaja@gmail.com

\section{Abstract}

Association between cerebral aneurysms and sellar tumors has been previously reported. Rupture of anterior circulation aneurysms during a transsphenoidal surgery causing massive subarachnoid hemorrhage (SAH) is uncommon, but rupture of a posterior circulation aneurysm is an infrequent event.

We present three cases of SAH secondary to rupture of an undetected posterior circulation aneurysm during transsphenoidal surgery to treat a sellar tumor. The common factor in these cases was the adverse outcome despite treatment.

The fatal outcome seen in all these cases questions whether to include a (magnetic resonance) MR angiography or (computed tomography) CT angiography during preoperative evaluation for sellar tumors in order to identify inadvertently associated aneurysms.

Categories: Neurology, General Surgery, Neurosurgery

Keywords: transsphenoidal surgery, posterior circulation aneurysm, ruptured cerebral aneurysm, subarachnoid hemorrhage, sellar tumor

\section{Introduction}

Pituitary tumors coexisting with cerebral aneurysms are a well-recognized association, but their frequency has not been definitively established.

Some studies report an incidence of $3.7 \%-7.4 \%$, with $97 \%$ of the aneurysms located in the anterior circulation [1-4]. The occurrence of subarachnoid hemorrhage (SAH) caused by the rupture of a coexisting cerebral aneurysm is quite unusual, and SAH caused by the inadvertent rupture of a posterior circulation aneurysm during transsphenoidal surgery is out of the ordinary [5-9].

This work presents a case series of three patients operated on for a sellar tumor through a transsphenoidal route as scheduled cases. In all three, an unexpected sudden neurologic impairment established the diagnosis of a massive SAH, and rupture of a non-detected posterior circulation aneurysm on preoperative imaging studies was shown to be the cause.

\section{Case Presentation}

\section{Case 1}

This 38-year-old male presented occasional galactorrhea for the past six years. He started experiencing progressive visual loss two years ago, leading to the ophthalmologist who referred the patient to neurosurgical evaluation because bitemporal hemianopsia was found. Prolactin levels (PRL) were 3430 $\mathrm{ng} / \mathrm{mL}$, and the magnetic resonance imaging (MRI) showed a sellar/suprasellar mass bulging into the prepontine cistern and involving both cavernous sinuses (Figure $1 \mathrm{~A}-1 \mathrm{C}$ ). Medical treatment with cabergoline lowered PRL to $68 \mathrm{ng} / \mathrm{mL}$, but with no shrinkage of the tumor and poor visual results, despite one year of pharmacologic treatment, making surgical removal necessary. 


\section{Cureus}

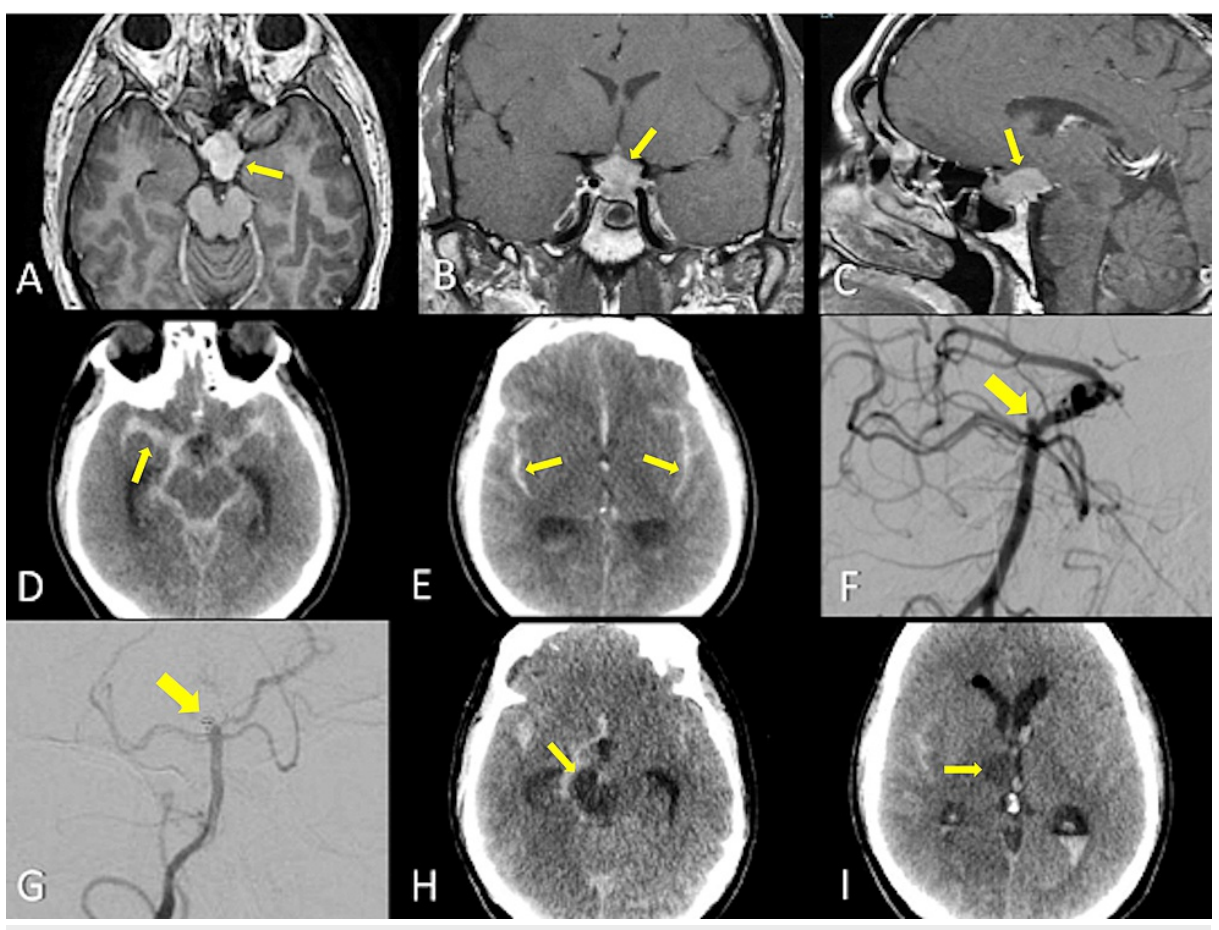

FIGURE 1: Case 1 representative MRI, CT, and DSA images.

(A-C) MRI shows a sellar tumor compatible with pituitary adenoma bulging into the mesencephalic cistern (arrows). (D,E) PO CT displaying a massive subarachnoid hemorrhage extending into the peri-mesencephalic cisterns (arrows). (F) A small basilar top aneurysm was discovered during the DSA (arrow). (G) The aneurysm was coiled for dome protection (arrow). ( $\mathrm{H}, \mathrm{I}) \mathrm{CT}$ at PO day 12. Low-density areas appeared at mesencephalic and thalamic areas (arrows).

MRI, magnetic resonance imaging; CT, computed tomography; DSA, digital subtraction angiography; $\mathrm{PO}$, postoperative.

During the transsphenoidal approach, a sudden diaphragma sellae descent with systolic hypertension at 240 $\mathrm{mm} \mathrm{Hg}$ and bradycardia prevented from continuing the procedure. A considerable pinkish secretion came out of the endotracheal tube, with a suspicion of pulmonary edema. Marked bilateral dilated pupils required an emergent unenhanced $\mathrm{CT}$, revealing a severe $\mathrm{SAH}$ centered on the peri-mesencephalic cistern with reflux into the ventricles and moderate ventricular enlargement (Figure 1D-1E). After inserting an intracranial pressure monitor and an external ventricular drain placement for cerebrospinal fluid diversion, a catheterbased angiography disclosed a small top basilar aneurysm, later managed with coiling (Figure 1F-1G).

The postoperative course was complicated by diabetes insipidus, right middle cerebral artery vasospasm seen on transcranial insonation, and delayed cerebral ischemia. Diffuse infarcts from the right midbrain, thalamus, and cerebral hemisphere finally lead to the patient's death on day 15 (Figure $1 \mathrm{H}-1 \mathrm{I}$ ).

\section{Case 2}

Six weeks before admission, this otherwise healthy 15 -year-old male teenager initially presented with ocular pain, visual acuity impairment, frontal pulsatile exertional headache, recent nausea, and vomiting. The eye examination found papilledema, and loss of visual acuity on both eyes, while abduction of the left eye was limited. The imaging workup revealed a $43 \times 27 \times 28 \mathrm{~mm}$ sellar and suprasellar tumor, most likely a craniopharyngioma with secondary hypogonadotropic hypogonadism (Figure $2 \mathrm{~A}-2 \mathrm{C}$ ). 


\section{Cureus}

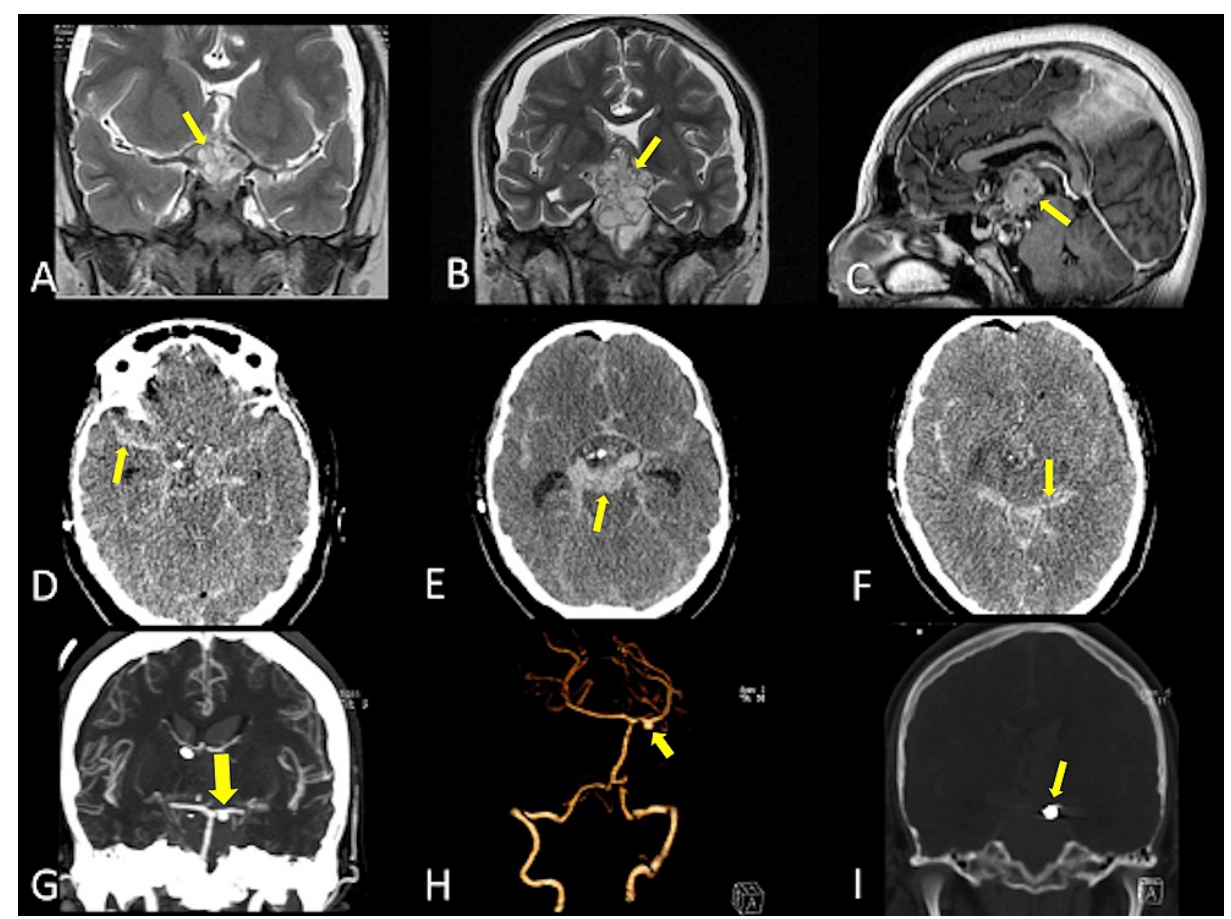

FIGURE 2: Case 2 representative MRI and CT angio images.

(A-C) MRI shows a suprasellar lesion extending upwards. A retrosellar extension of the tumor is visible (arrows). (D-F) During surgery, severe bleeding was noticed and controlled. CT scan shows a generalized SAH with thick blood clots at the prepontine cistern (arrows). (G,H) CT angio shows a right small P1 aneurysm (arrows). (I) The aneurysm was treated by coiling (arrow).

$\mathrm{CT}$, computed tomography; MRI, magnetic resonance imaging; SAH, subarachnoid hemorrhage.

The patient was uneventfully managed with a right frontal ventriculoperitoneal (VP) shunt for obstructive hydrocephalus and a transsphenoidal resection. Heavy bleeding from the sellar area was controlled during surgery, but a postoperative CT revealed a Fisher $4 \mathrm{SAH}$ (Figure $2 D-2 F$ ). An external ventricular drain replaced the VP shunt. A CT angiography showed a left P1 aneurysm $(5.4 \times 5.3 \mathrm{~mm}$ with a neck of $3.8 \mathrm{~mm})$ that was subsequently coiled with obliteration of $85 \%$ (Figure $2 G-2 I$ ). Cerebral vasospasm in both anterior cerebral arteries and the left posterior cerebral artery marked a poor evolution, leading to anisocoria, bilateral fixed pupils, and loss of brain stem reflexes. The transcranial Doppler with a reverberant pattern confirmed the diagnosis of brain death, and the patient died two days later.

\section{Case 3}

A progressive visual impairment led this 45 -year-old male to the ophthalmologist, who referred the patient with a typical chiasmatic visual field defect. The MRI study revealed a sellar lesion $1.8 \times 1.0 \times 1.5 \mathrm{~cm}$ in dimensions (Figure 3A-3C). As a nonfunctioning pituitary adenoma, he was taken to a transsphenoidal removal of the lesion with an unremarkable procedure. During emergence from anesthesia, the patient suddenly deteriorated with bilateral dilated and fixed pupils. A Fisher grade $3 \mathrm{SAH}$ with obstructive hydrocephalus was shown by an unenhanced CT (Figure 3D-3F). After ventriculostomy placement, a 3-mm basilar aneurysm (Figure 3G-3H) at the superior cerebellar segment junction was diagnosed at digital subtraction angiography (DSA) and subsequently coiled for dome protection (Figure 31). He progressively deteriorated until brain death and a final, fatal outcome. 


\section{Cureus}

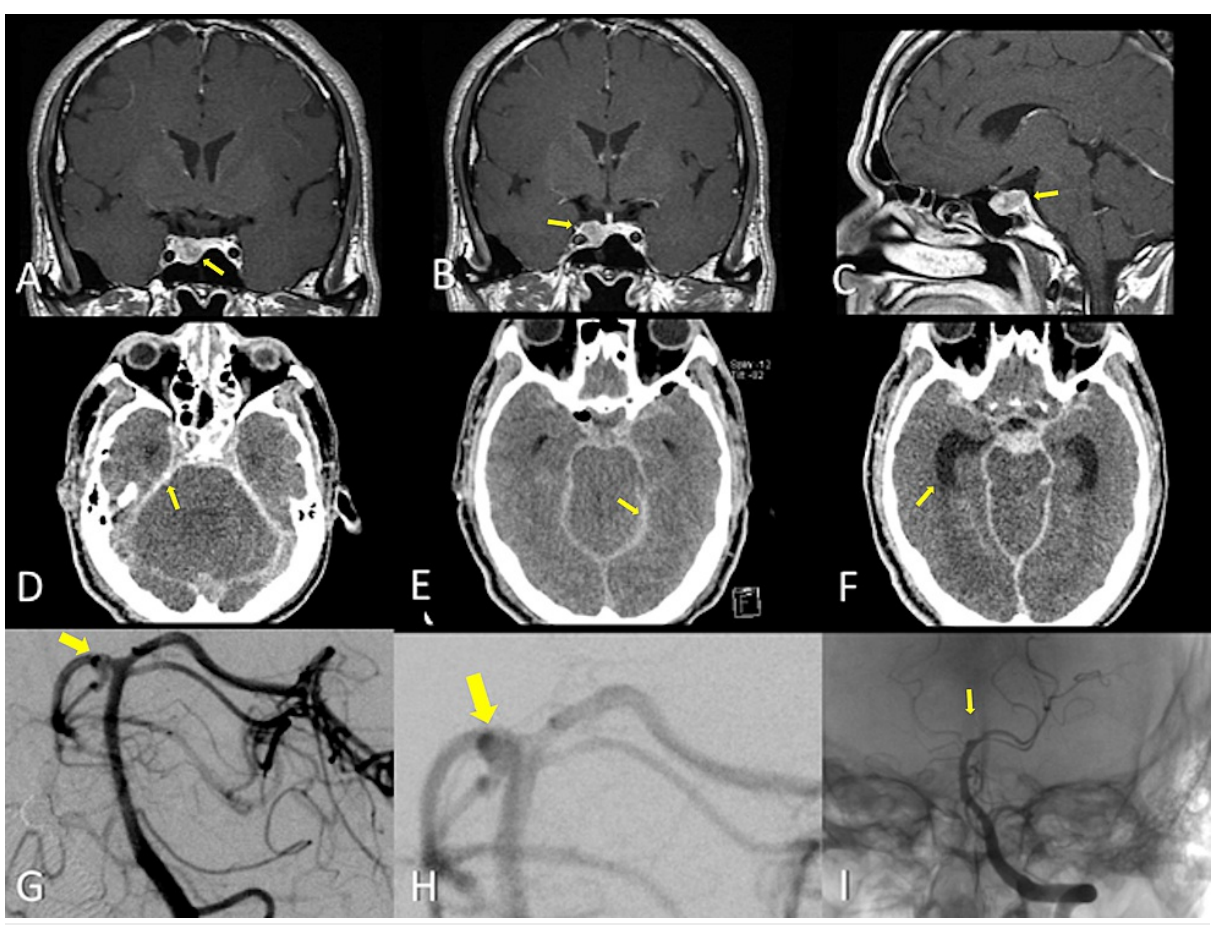

FIGURE 3: Case 3 representative MRI, CT, and DSA images.

(A-C) MRI shows a sellar tumor without retrosellar extension (arrows). (D,E) PO CT scan showing a massive SAH filling all basal cisterns (arrows). (F) Early ventricular enlargement is seen (arrow). (G,H) A small basilar-superior cerebellar artery segment aneurysm is seen (arrow). (I) The patient was coiled for dome protection (arrow).

$\mathrm{CT}$, computed tomography; MRI, magnetic resonance imaging; DSA, digital subtraction angiography; PO, postoperative; $\mathrm{SAH}$, subarachnoid hemorrhage.

\section{Discussion}

Transsphenoidal surgery is one of the procedures of choice to remove pituitary adenomas and other sellar tumors, with mortality and morbidity rates below $0.5 \%$ and $1.5 \%$, respectively $[10]$. Commonly reported complications are cerebrospinal fluid fistulas, pneumocephalus, neuroinfection, intracavernous carotid artery pseudoaneurysm formation, and traumatic SAH.

Cerebral aneurysms coexist with pituitary tumors more often than expected, and their presence on the major arteries adjacent to the pituitary and suprasellar tumors entails considerable risks, particularly when the aneurysm is closer to the operative field $[1,11-13]$. They are commonly detected at the imaging workup stage in preoperative MRI. It is, therefore, possible to plan the treatment of both lesions, separate or during the same procedure, particularly when the aneurysm is the area and is accessible from the same surgical approach [3]. Besides serendipity, another reason for explaining this association is the direct mechanical effect of sellar tumors on the vasculature, direct infiltration by tumor, and growth hormone production leading to arteriosclerosis, hypertension, and diabetes $[1,2,8]$.

Previous reports locate most of these aneurysms in the anterior circulation, particularly in the anterior communicating or the internal carotid arteries, possibly because these arteries are related to the blood supply to the sellar region $[4-6,11,13]$. In one of these series, the aneurysm's location was posterior communicating segment of the internal carotid artery in $48 \%$, ophthalmic segment in $19 \%$, and carotid bifurcation in $13 \%$ [12]. In another study, $60 \%$ of the aneurysms were near the parasellar region and $40 \%$ away from it [2]. In these patients, the risk of aneurysmal rupture is always present. Some moments are particularly at risk: emergence from anesthesia or intraoperative manipulation to achieve a better tumor removal, direct mechanical impact, or intraoperative hemodynamic changes.

On the contrary, the occurrence with posterior circulation aneurysms is unusual and has been reported only twice. One was an autopsy case where a pituitary adenoma was associated with a giant vertebrobasilar aneurysm, and the other described a fatal SAH from a basilar apex aneurysm in the postoperative period $[7,14]$. Rupture of a posterior circulation aneurysm during craniopharyngioma removal has never been reported. These three cases are thus the largest series reporting the association of an undetected aneurysm of the posterior circulation associated with a sellar tumor. In all three cases, postoperative imaging documented a small posterior circulation aneurysm (top basilar, basilar artery-superior cerebellar artery segment, and P1 segment). Preoperative workup never disclosed clearly the presence of the aneurysm, and 
in contrast with reported cases regarding intraoperative rupture of anterior circulation aneurysms, all three patients had a fatal outcome $[5,6,15,16]$. Hemodynamic changes are less likely to occur in the posterior circulation while removing a pituitary adenoma or a craniopharyngioma compared with the anterior circulation. However, a retrosellar extension might be a risk factor for mechanical injury during tumor excision, even when the resection is made without incurring any extraordinary surgical maneuver.

Despite its rarity, postoperative SAH can have dismal consequences, as shown in this series, and it might deserve further consideration. Given its technical feasibility during the preoperative imaging workup without significantly increasing healthcare costs or scanning time, we wonder if complementary preoperative MR angiography or CT angiography should be performed, mainly when there is a mechanical involvement or closeness to vascular structures. Additionally, DSA should be mandatory when an associated aneurysm is detected preoperatively. In such cases, a better plan can be done including the dome protection through an endovascular route before the transsphenoidal surgery to avoid an eventual rupture. Another option is the treatment of both, the aneurysm and the adenoma, through a transcranial route at the same time.

Even when anterior circulation aneurysms are more commonly associated with pituitary adenomas (especially growth hormone-secreting tumors), posterior circulation aneurysms are not common in such situations. Focused preoperative imaging might be a way to prevent unforeseen catastrophic events. Moreover, this point remains controversial and demands further investigations.

\section{Conclusions}

The coexistence of sellar and suprasellar tumors and posterior circulation aneurysms are rare. The occurrence of SAH after tumor removal is exceedingly exceptional and has rarely been reported. However, the fatal outcome of this unexpected event in an otherwise nonmortal disease deserves further study to determine if these cases could benefit from a more extensive workup vascular imaging in the preoperative stage.

\section{Additional Information \\ Disclosures}

Human subjects: Consent was obtained or waived by all participants in this study. Conflicts of interest: In compliance with the ICMJE uniform disclosure form, all authors declare the following: Payment/services info: All authors have declared that no financial support was received from any organization for the submitted work. Financial relationships: All authors have declared that they have no financial relationships at present or within the previous three years with any organizations that might have an interest in the submitted work. Other relationships: All authors have declared that there are no other relationships or activities that could appear to have influenced the submitted work.

\section{References}

1. Jakubowski J, Kendall B: Coincidental aneurysms with tumours of pituitary origin . J Neurol Neurosurg Psychiatry. 1978, 41:972-9. 10.1136/jnnp.41.11.972

2. Pant B, Arita K, Kurisu K, Tominaga A, Eguchi K, Uozumi T: Incidence of intracranial aneurysm associated with pituitary adenoma. Neurosurg Rev. 1997, 20:13-7. 10.1007/BF01390519

3. Revuelta R, Arriada-Mendicoa N, Ramirez-Alba J, Soto-Hernandez JL: Simultaneous treatment of a pituitary adenoma and an internal carotid artery aneurysm through a supraorbital keyhole approach. Minim Invasive Neurosurg. 2002, 45:109-11. 10.1055/s-2002-32488

4. Yu K, Herwadkar A, Kearney T, Gnanalingham KK: Pituitary adenoma and incidental superior hypophyseal aneurysm. Br J Neurosurg. 2011, 25:432-3. 10.3109/02688697.2010.509521

5. Akutsu N, Hosoda K, Ohta K, Tanaka H, Taniguchi M, Kohmura E: Subarachnoid hemorrhage due to rupture of an intracavernous carotid artery aneurysm coexisting with a prolactinoma under cabergoline treatment. J Neurol Surg Rep. 2014, 75 :e73-6. 10.1055/s-0033-1364166

6. Almeida Silva JM, Campos RR, Souza RR, Sette Dos Santos ME, Aguiar GB: Spontaneous subarachnoid haemorrhage from rupture of an anterior communicating artery aneurysm in a patient with pituitary macroadenoma. Neurocirugia (Astur). 2014, 25:81-5. 10.1016/j.neucir.2013.03.005

7. Habibi Z, Miri SM, Sheikhrezaei A: Pituitary macroadenoma coexistent with a posterior circulation aneurysm leading to subarachnoidal hemorrhage during transsphenoidal surgery. Turk Neurosurg. 2015, 25:469-74. 10.5137/1019-5149.JTN.8314-13.0

8. Khalsa SS, Hollon TC, Shastri R, Trobe JD, Gemmete JJ, Pandey AS: Spontaneous subarachnoid hemorrhage due to ruptured cavernous internal carotid artery aneurysm after medical prolactinoma treatment. J Neurointerv Surg. 2017, 9:e9. 10.1136/neurintsurg-2016-012446.rep

9. Matsuno A, Yoshida S, Basugi N, Itoh S, Tanaka J: Severe subarachnoid hemorrhage during transsphenoidal surgery for pituitary adenoma. Surg Neurol. 1993, 39:276-8. 10.1016/0090-3019(93)90004-k

10. Laws ER, Jane JA Jr: Neurosurgical approach to treating pituitary adenomas . Growth Horm IGF Res. 2005, 15 Suppl A:S36-41. 10.1016/j.ghir.2005.06.008

11. Tsuchida T, Tanaka R, Yokoyama M, Sato H: Rupture of anterior communicating artery aneurysm during transsphenoidal surgery for pituitary adenoma. Surg Neurol. 1983, 20:67-70. 10.1016/0090-3019(83)90110-6

12. Wang CS, Yeh TC, Wu TC, Yeh CH: Pituitary macroadenoma co-existent with supraclinoid internal carotid artery cerebral aneurysm: a case report and review of the literature. Cases J. 2009, 2:6459. 10.4076/1757- 


\section{Cureus}

1626-2-6459

13. Xu K, Yuan Y, Zhou J, Yu J: Pituitary adenoma apoplexy caused by rupture of an anterior communicating artery aneurysm: case report and literature review. World J Surg Oncol. 2015, 13:228. 10.1186/s12957-0150653-z

14. Nadjem H, Müller K, Riede U, Pollak S: Rare coincidence of a giant aneurysm of the basilar artery with a macroadenoma of the pituitary gland in a forensic autopsy. Forensic Sci Int. 2007, 172:191-5. 10.1016/j.forsciint.2006.08.012

15. Song RX, Wang DK, Wang Z, Wang ZW, Wang SX, Wei GX, Li XG: Coexistence of aneurysmal subarachnoid hemorrhage and surgically identified pituitary apoplexy: a case report and review of the literature. J Med Case Rep. 2014, 8:166. 10.1186/1752-1947-8-166

16. Goyal N, Basheer N, Suri A, Mahapatra AK: Subarachnoid hemorrhage after transsphenoidal surgery for pituitary adenoma: a case report and review of literature. Neurol India. 2012, 60:337-8. 10.4103/00283886.98532 